\title{
Avaliação de unidades portuárias brasileiras com análise envoltória de dados e o método multicritério ordinal de Copeland
}

\author{
Ana Paula dos Santos Rubem¹, Luana Carneiro Brandão² e João Carlos Correia Baptista Soares de Mello 3
}

\begin{abstract}
Resumo: Como o tipo de carga influi diretamente na infraestrutura necessária à sua movimentação, algumas unidades portuárias buscam a especialização ou diversificação das atividades. Este trabalho utiliza a Análise Envoltória de Dados e o método multicritério ordinal de Copeland para avaliar a importância relativa de cada unidade do setor portuário brasileiro para o comércio nacional, dentro do respectivo segmento em que cada uma delas atua, comparando-se ambas as técnicas. Dada a não homogeneidade, as unidades são distribuídas em oito clusters, conforme a natureza da carga que operam. Como tal segregação é imediata, não é usada nenhuma ferramenta matemática para este fim. Em seguida, são aplicadas as duas abordagens supracitadas de apoio à decisão. No primeiro caso, é usado um modelo com input unitário, em que o input representa a própria existência da unidade portuária; além disto, para aumentar o poder de discriminação, utiliza-se o índice de eficiência composto normalizado no lugar do índice padrão. Os resultados indicam que as ordenações Copeland e do índice composto são bastante semelhantes. O presente trabalho também esclarece as diferenças entre os resultados de DEA com input unitário e de métodos multicritérios ordinais, indicando o modelo mais adequado em cada situação.
\end{abstract}

Palavras-chave: Avaliação portuária, Análise Envoltoria de Dados (DEA), Método Ordinal de Copeland. Abstract: As the cargo type directly influences the required infrastructure to its handling, some port units seek the special-
ization or diversification of its activities. This study applies Data Envelopment Analysis and, for comparative purposes,
Copeland's ordinal method to evaluate the relative importance of each unit in the Brazilian port sector for national trade
within the business sector each one operates. Given the non-homogeneity, we divide the units in eight clusters, depending on
the nature of the cargo handled. As this segregation is straightforward, we do not use any mathematical tool for this purpose.
Then, we apply the two above-mentioned decision-aid approaches. In the first case, we use a unitary input model, in which
the input is the very existence of the port unit; in addition, to increase the discrimination, we use the normalized composite
efficiency index instead of the standard. The results indicate that the Copeland and the composite index rankings are quite
similar. This study also highlights the differences between unitary input DEA models and ordinal methods, with regard to
their results, indicating the most adequate model in each type of situation.

Keywords: Port Evaluation, Data Envelopment Analysis (DEA), Copeland's Ordinal Method.

\section{INTRODUÇÃO}

É inegável a relevância do setor portuário para o desenvolvimento econômico. Por isso, há vários estudos que avaliam o desempenho portuário e permitem uma visão geral do setor em diversos países. Contudo, dadas as características distintas de cada unidade portuária em termos de insumos e produção, que decorrem, principalmente, da natureza diferenciada da carga operada, muitos optam pela análise de unidades especializadas.

Neste artigo, como em Bertoloto e Soares de Mello (2011) e Acosta et al. (2011), busca-se uma análise mais ampla, ainda que restrita ao transporte de mercadorias. O objetivo é permitir a avaliação de unidades portuárias com perfis diversificados, em razão dos diferentes tipos de carga que operam, a fim de identificar a importância de cada unidade para o comércio do país. Para tanto, é conduzido um

\footnotetext{
1 Ana Paula dos Santos Rubem, Universidade Federal Fluminense. (anarubem@id.uff.br)

2 Luana Carneiro Brandão, Universidade Federal Fluminense. (luanabrandao@id.uff.br)

3 João Carlos Correia Baptista Soares de Mello, Departamento de Engenharia de Produção -Universidade Federal Fluminense.

(jcsmello@producao.uff.br)
}

Manuscrito recebido em 24/08/2015 e aprovado para publicação em 04/09/2015.

Este artigo é parte de TRANSPORTES v. 23, n. 4, 2015. ISSN: 2237-1346

(online). DOI:10.14295/transportes.v23i4.988

TRANSPORTES v. 23, n. 4 (2015), p. 31-41 estudo de caso sobre o desempenho das diversas unidades que compõem o complexo portuário brasileiro, considerando o volume de carga movimentado, segundo sua natureza, bem como o número de atracações.

Diferentemente dos trabalhos supracitados, neste estudo, além de serem utilizados outputs que são específicos a cada tipo de carga, as unidades não especializadas serão divididas em grupos distintos, conforme a combinação de carga operada. Ademais, ao invés de enfatizar o papel da autoridade portuária, aqui, a análise restringir-se-á à atividade-fim (i.e., à movimentação de carga em si); e não à gestão de recursos relativos à infraestrutura, mão de obra ou capital.

Desse modo, dada a não homogeneidade das unidades que compõem o setor portuário brasileiro, elas serão agrupadas, conforme a natureza da carga movimentada. Como tal agrupamento é imediato, não será usado nenhum ferramental matemático para este fim. Em seguida, são aplicadas duas abordagens de apoio à decisão: a Análise Envoltória de Dados (DEA, de Data Envelopment Analysis: Charnes et al., 1978), utilizando-se a modelagem de input unitário proposta em Lovell e Pastor (1999), e o método multicritério ordinal de Copeland (1951), separadamente para cada grupo de unidades homogêneas.

O objetivo do emprego de ambas as técnicas de apoio à decisão é avaliar o desempenho das unidades portuárias dentro do segmento ao qual pertencem, com base nas duas abordagens. Isso porque tal formato de análise de dados, 
restrito ao desempenho das unidades, pode se assemelhar a métodos multicritérios ordinais, apesar das diferenças entre as abordagens.

A Seção 2 descreve os métodos utilizados; a Seção 3 contém uma breve revisão sobre o uso de DEA em avaliação portuária; a Seção 4 descreve o estudo de caso; a Seção 5 apresenta e discute os resultados. Por fim, a Seção 6 contempla algumas considerações finais e sugestões para trabalhos futuros.

\section{REFERENCIAL TEÓRICO}

\subsection{DEA: Análise Envoltória de Dados}

DEA (Charnes et al., 1978) é uma abordagem nãoparamétrica, baseada em programação matemática, cujo objetivo é medir o desempenho de unidades (ou DMUs, de Decision Making Units), que são otimizadas individualmente, comparando os recursos utilizados (inputs) e as quantidades produzidas (outputs). O conjunto de DMUs em análise deve operar sob a mesma tecnologia, usando os mesmos tipos de inputs e de outputs (pressuposto de homogeneidade). O resultado é a construção de uma fronteira Pareto eficiente, em que as DMUs nela situadas apresentam índice de $100 \%$. Em geral, os modelos dispõem de formulações duais (envelope e multiplicadores), havendo duas orientações radiais possíveis: a inputs, que busca minimizar os recursos utilizados mantidos inalterados os níveis de produção; e a outputs, que implica no aumento da produção sem alterar as quantidades de insumos utilizadas.

Como o objetivo é analisar o desempenho das unidades, não relativizado pelos recursos disponíveis, para posterior confrontação com os resultados do método de Copeland, optou-se pelo modelo com input único, constante e unitário, o qual denota a própria existência da DMU. Na realidade, o input unitário é utilizado como simples artifício numérico, uma vez que um modelo sem inputs gera inconsistências matemáticas. Neste trabalho, a abordagem usada é a de Lovell e Pastor (1999), em que os modelos CCR (Charnes et al., 1978) e BCC (Banker et al., 1984) orientado a outputs são equivalentes. O modelo com input unitário, orientado a outputs, na formulação do envelope é dado por:

$$
\begin{aligned}
& \operatorname{Max} h_{o} \\
& \text { sujeito a } \\
& \sum_{k=1}^{n} \lambda_{k} y_{j k} \geq h_{o} y_{j o}, \forall j \mathrm{k} \\
& \sum_{k=1}^{n} \lambda_{k} \leq 1 \\
& \lambda_{k} \geq 0, \forall k
\end{aligned}
$$

onde $h_{o}$ é o inverso da eficiência da DMU em análise $\left(\mathrm{DMU}_{\mathrm{o}}\right) ; y_{j k}$ é o $j$-ésimo output $(j=1, \ldots, s)$ da $\mathrm{DMU}_{\mathrm{k}}$ $(k=1, \ldots, n)$; e $\left\{\lambda_{k}\right\}$ é a contribuição de cada DMU na formação do alvo da $\mathrm{DMU}_{\mathrm{o}}$. Esse modelo pode ser interpretado como um modelo multicritério aditivo em que as alternati- vas (DMUs) atribuem pesos aos critérios (outputs), ignorando qualquer juízo de valor do decisor. Sob tais condições, pode-se afirmar que o modelo DEA é usado mais como uma ferramenta multicritério, do que como um instrumento para o cálculo de uma medida de eficiência (Gomes et al., 2012). Para maiores informações sobre esse tipo de modelo, consulte Cook e Zhu (2005).

\subsection{Método de Copeland}

Copeland (1951) propôs um método multicritério ordinal que considera apenas a ordenação das alternativas em cada critério, e não seus valores exatos. Para cada alternativa, somam-se as diferenças em cada critério entre o número de alternativas melhores e o número de alternativas piores. Por fim, ordenam-se as alternativas por essa soma. Como qualquer método multicritério não ditatorial, o de Copeland não é perfeito, pois não atende a todos os axiomas de Arrow (1951): universalidade, transitividade, unanimidade e independência das alternativas irrelevantes.

Neste estudo, a escolha desse método se deu por representar uma proposta intermediária aos métodos de Condorcet e de Borda (Barba-Romero e Pomerol, 1997), também destinados à problemática da ordenação multicritério. De fato, na ausência de intransitividade, Copeland fornece o mesmo resultado que Condorcet; mas, diante de ciclos de intransitividade, é menos sensível às alternativas irrelevantes que Borda. Os métodos multicritério ordinais são muito usados em esportes (e.g., Soares de Mello et al., 2005), e sua principal vantagem é a simplicidade. Para uma discussão mais abrangente, veja Barba-Romero e Pomerol (1997).

\section{DEA APLICADA AO SETOR PORTUÁRIO}

Roll e Hayuth (1993) conduziram um dos primeiros estudos a aplicar DEA na avaliação de desempenho portuário. Enfatizando aspectos financeiros, Martinez-Budria et al. (1999) analisaram 26 portos espanhóis. Com foco operacional, Tongzon (2001) avaliou dezesseis portos em diferentes países. Valentine e Gray (2001), Itoh (2002), Turner et al. (2004) restringiram-se a terminais de contêiner. Outras aplicações podem ser encontradas em Barros e Athanassiou (2004), Barros (2006), Rios e Maçada (2006) e Lozano e Villa (2009).

Em relação aos portos brasileiros, Pires et al. (2009) analisaram unidades de carregamento de minério de ferro. Bertoloto e Soares de Mello (2011) avaliaram 47 unidades marítimas com perfis distintos, combinando uma técnica de homogeneização a um modelo com dois inputs estruturais (extensão dos berços e calado máximo) e um único output (total de cargas movimentadas). Com variáveis similares, Acosta et al. (2011) analisaram 27 portos brasileiros não especializados. Caillaux et al. (2011) usaram um modelo com output unitário na seleção de porto para transporte de contêineres, comparando-o aos métodos de Copeland e composição probabilística. Cortez et al. (2013) avaliaram o desempenho gerencial de oito autoridades portuárias. Além dos diferenciais apontados na seção anterior, este estudo se diferencia dos supracitados, à exceção de Caillaux et al. (2011), por usar uma abordagem com input unitário. 


\section{ESTUDO DE CASO}

\subsection{Escopo, variáveis e base de dados}

Neste artigo, as unidades analisadas são os portos públicos, terminais de uso privado (TUPs) e estações de transbordo de carga (ETCs) do setor portuário (marítimo e fluvial) brasileiro, em atividade no ano de 2012, destinados ao transporte de mercadorias. Busca-se avaliá-los, quanto à movimentação de carga, considerando o input unitário e os outputs: número de atracações (em todos os clusters); quantidade movimentada de carga em contêineres (em TEUs, de Twenty-Foot Equivalent Unit ou unidade equivalente a 20 pés); tonelagem movimentada de carga solta; tonelagem movimentada de carga em granel líquido; e a tonelagem de carga movimentada em granel sólido. O output número de atracações é usado na avaliação das unidades pertencentes a todos os clusters; enquanto os demais outputs variam conforme o tipo de carga operado pelas unidades pertencentes ao cluster em questão, de modo a não haver outputs nulos.

Optou-se pelo input unitário porque, diferentemente da maioria dos trabalhos existentes na literatura que avaliam a eficiência de cada unidade portuária em relação aos recursos utilizados (e.g., extensão de cais, número de berços, guindastes, mão-de-obra), o objetivo aqui é analisar o "produto" final das unidades portuárias, sem relativizá-lo pelos recursos disponíveis. Assim, os outputs definidos para o modelo denotam o "produto" da atividade portuária, isto é, a movimentação dos diferentes tipos de carga. Contudo, no modelo aqui proposto, o número de atracações também é considerado como output para que unidades de baixo calado, e, portanto, restritas à movimentação de pouca tonelagem a cada atracação, possam compensar tal limitação, desde que mantenham um nível elevado de atracações. Desse modo, pressupõem-se aqui que um grande número de atracações denota um bom desempenho, a despeito do volume total de carga movimentado.

Na movimentação de carga em contêineres, TEU é a medida padrão usada para calcular a capacidade de transporte dos navios porta-contêineres (e.g., um contêiner de 40 pés equivale a dois TEUs). A carga geral solta denota cargas acondicionadas em diversas formas e dimensões (e.g., arroz em sacas, blocos de granitos, veículos). A carga em granel líquido se refere à carga líquida transportada nos porões, sem embalagem, em grandes quantidades, movimentada em dutos, por meio de bombas (e.g., petróleo, óleos vegetais, suco de laranja). A carga em granel sólido denota a carga seca fragmentada, constituída de sólidos transportados nos porões (e.g., trigo, milho, minério de ferro, farelos, soja).

Os dados apresentados na Tabela 1 referem-se a 133 unidades portuárias que movimentaram carga em 2012, sendo 33 portos públicos, 97 TUPs e 3 ETCs. Os mesmos foram obtidos no site da Agência Nacional de Transporte Aquaviário (ANTAQ), por meio do Sistema de Informações Gerencias Acesso Público 1. Optou-se pelo ano de 2012, pois os dados de 2013 podiam sofrer correções e atualizações em data posterior à elaboração deste artigo.

Tabela 1. Outputs observados para cada unidade produtiva (porto/TUP), em 2012

\begin{tabular}{|c|c|c|c|c|c|c|c|}
\hline Porto/TUPs & $\boldsymbol{U} \boldsymbol{F}$ & $D M U$ & $\begin{array}{l}\text { Núm. de } \\
\text { Atracações }\end{array}$ & Qtde TEUs & $\begin{array}{l}\text { Carga Solta } \\
(t)\end{array}$ & \begin{tabular}{|l|} 
Granel \\
Sólido $(t)$ \\
\end{tabular} & $\begin{array}{l}\text { Granel } \\
\text { Líquido(t) }\end{array}$ \\
\hline ANGRA DOS REIS & RJ & 1 & 83 & 0 & 23136 & 31998 & 31949 \\
\hline ANTONINA & PR & 2 & 92 & 0 & 99454 & 1161547 & 0 \\
\hline ARATU & BA & 3 & 601 & 0 & 0 & 1692382 & 4110724 \\
\hline AREIA BRANCA & $\mathrm{RN}$ & 4 & 1026 & 0 & 0 & 1995945 & 0 \\
\hline BELÉM & PA & 5 & 1174 & 23121 & 212388 & 590467 & 2165336 \\
\hline CABEDELO & $\mathrm{PB}$ & 6 & 147 & 0 & 60546 & 1102035 & 744857 \\
\hline ESTRELA & $\mathrm{RS}$ & 7 & 3 & 0 & 0 & 7244 & 0 \\
\hline ETC BERTOLINI SANTANA & $\mathrm{AP}$ & 8 & 154 & 0 & 146532 & 0 & 0 \\
\hline ETC ITACAL & $\mathrm{AM}$ & 9 & 14 & 0 & 0 & 16 & 0 \\
\hline ETC PORTO MURTINHO & MS & 10 & 3 & 0 & 0 & 3974 & 0 \\
\hline FORNO & $\mathrm{RJ}$ & 11 & 20 & 6 & 6160 & 153828 & 0 \\
\hline FORTALEZA & $\mathrm{CE}$ & 12 & 569 & 62969 & 215948 & 1250932 & 2381987 \\
\hline ILHÉUS & $\mathrm{BA}$ & 13 & 47 & 0 & 73160 & 386781 & 0 \\
\hline IMBITUBA & $\mathrm{SC}$ & 14 & 195 & 20984 & 137005 & 1549205 & 106378 \\
\hline ITAGUAÍ (SEPETIBA) & $\mathrm{RJ}$ & 15 & 845 & 332195 & 130567 & 52795311 & 0 \\
\hline ITAJAÍ & $\mathrm{SC}$ & 16 & 300 & 386537 & 0 & 0 & 0 \\
\hline ITAQUI & MA & 17 & 795 & 8498 & 158338 & 7896585 & 7608370 \\
\hline MACAPÁ & $\mathrm{AP}$ & 18 & 340 & 0 & 42548 & 767010 & 693311 \\
\hline MACEIÓ & $\mathrm{AL}$ & 19 & 357 & 0 & 106580 & 1986580 & 907712 \\
\hline NATAL & $\mathrm{RN}$ & 20 & 89 & 28932 & 39361 & 153323 & 154 \\
\hline NITERÓI & $\mathrm{RJ}$ & 21 & 392 & 0 & 62010 & 0 & 64 \\
\hline PARANAGUÁ & PR & 22 & 2141 & 743830 & 874146 & 29874836 & 3120028 \\
\hline PELOTAS & RS & 23 & 6 & 0 & 0 & 13331 & 0 \\
\hline PORTO ALEGRE & RS & 24 & 186 & 0 & 5847 & 898802 & 0 \\
\hline PORTO VELHO & RO & 25 & 1864 & 180 & 464445 & 2805545 & 435 \\
\hline RECIFE & $\mathrm{PE}$ & 26 & 354 & 3768 & 197084 & 1463016 & 19396 \\
\hline RIO DE JANEIRO & RJ & 27 & 1415 & 437454 & 777671 & 1309054 & 22006 \\
\hline RIO GRANDE & $\mathrm{RS}$ & 28 & 2406 & 612618 & 880543 & 7181563 & 2900371 \\
\hline SALVADOR & BA & 29 & 550 & 251566 & 227767 & 316763 & 66954 \\
\hline SANTARÉM & PA & 30 & 1604 & 4503 & 40393 & 3210575 & 130863 \\
\hline SANTOS & SP & 31 & 5355 & 3172335 & 3311703 & 43949695 & 12206577 \\
\hline SÃO FRANCISCO DO SUL & $\mathrm{SC}$ & 32 & 635 & 118264 & 2411912 & 7011808 & 158000 \\
\hline SÃO SEBASTIÃO & SP & 33 & 109 & 747 & 276095 & 604213 & 0 \\
\hline
\end{tabular}


RUBEM, A.P.S.; BRANDÃO, L.C.; SOARES DE MELLO, J.C.C.B.

Tabela 1. Outputs observados para cada unidade produtiva (porto/TUP), em 2012

\begin{tabular}{|c|c|c|c|c|c|c|c|}
\hline Porto/TUPs & $\boldsymbol{U F}$ & $D M U$ & $\begin{array}{l}\text { Núm. de } \\
\text { Atracações }\end{array}$ & Qtde TEUs & $\begin{array}{l}\text { Carga Solta } \\
(t)\end{array}$ & $\begin{array}{l}\text { Granel } \\
\text { Sólido }(t)\end{array}$ & $\begin{array}{l}\text { Granel } \\
\text { Líquido(t) }\end{array}$ \\
\hline SUAPE & $\mathrm{PE}$ & 34 & 1288 & 404590 & 178456 & 623644 & 5672689 \\
\hline TUP AGROPALMA & PA & 35 & 138 & 0 & 0 & 0 & 201296 \\
\hline TUP ALMIRANTE BARROSO & $\mathrm{SP}$ & 36 & 616 & 0 & 0 & 0 & 50541216 \\
\hline TUP ALMIRANTE MAXIMIANO & & & & & & & \\
\hline DA FONSECA & RJ & 37 & 253 & 0 & 0 & 430886 & 36610276 \\
\hline TUP ALMIRANTE SOARES DUTRA & RS & 38 & 193 & 0 & 0 & 0 & 11060986 \\
\hline TUP ALMIRANTE TAMANDARÉ & & & & & & & \\
\hline (ILHA D’ÁGUA) & RJ & 39 & 1355 & 0 & 0 & 629886 & 13169037 \\
\hline TUP ALUMAR & MA & 40 & 326 & 0 & 0 & 11996074 & 778158 \\
\hline TUP BELMONTE & $\mathrm{RO}$ & 41 & 196 & 0 & 122329 & 0 & 0 \\
\hline TUP BERTOLINI BELÉM & PA & 42 & 888 & 0 & 1055306 & 0 & 0 \\
\hline TUP BERTOLINI SANTARÉM & PA & 43 & 737 & 0 & 142373 & 0 & 0 \\
\hline TUP BIANCHINI & RS & 44 & 428 & 0 & 0 & 4794471 & 295656 \\
\hline TUP BRASFELS & RJ & 45 & 11 & 0 & 18210 & 0 & 0 \\
\hline TUP BRASKEM ALAGOAS & AL & 47 & 84 & 0 & 0 & 0 & 1020067 \\
\hline TUP CAIMA & $\mathrm{RO}$ & 48 & 98 & 0 & 80197 & 0 & 0 \\
\hline TUP CARGILL AGRICOLA & $\mathrm{RO}$ & 49 & 530 & 0 & 0 & 1179309 & 0 \\
\hline TUP CARMÓPOLIS & SE & 50 & 102 & 0 & 0 & 0 & 2744668 \\
\hline TUP CATTALINI & PR & 51 & 148 & 0 & 0 & 0 & 1562447 \\
\hline TUP CAULIM DA AMAZÔNIA & PA & 52 & 26 & 0 & 18803 & 221164 & 40566 \\
\hline TUP CEVAL & RS & 53 & 87 & 0 & 0 & 1009386 & 148466 \\
\hline TUP CHIBATÃO & $\mathrm{AM}$ & 54 & 170 & 273559 & 0 & 0 & 0 \\
\hline TUP CHIBATÃO 2 & $\mathrm{AM}$ & 55 & 1477 & 0 & 883085 & 0 & 0 \\
\hline TUP CIMBAGÉ & $\mathrm{RS}$ & 56 & 189 & 0 & 0 & 408112 & 0 \\
\hline TUP CIMENTO VENCEMOS & $\mathrm{AM}$ & 57 & 25 & 0 & 0 & 327810 & 0 \\
\hline TUP CMPC GUAÍBA & RS & 58 & 152 & 0 & 304520 & 0 & 0 \\
\hline TUP COPELMI & RS & 59 & 207 & 0 & 0 & 264684 & 0 \\
\hline TUP COTEGIPE & BA & 60 & 84 & 0 & 0 & 3205036 & 0 \\
\hline TUP CVRD PRAIA MOLE & ES & 61 & 175 & 0 & 0 & 10088116 & 0 \\
\hline GUANABARA & RJ & 63 & 46 & 0 & 0 & 0 & 2264524 \\
\hline TUP DNP - BASE DE DISTRIBUIÇÃO & & & & & & & \\
\hline SECUNDÁRIA DE SANTARÉM & PA & 64 & 33 & 0 & 0 & 478 & 22264 \\
\hline TUP DOW BRASIL GUARUJÁ & $\mathrm{SP}$ & 65 & 106 & 0 & 0 & 0 & 711672 \\
\hline TUP DOW ARATU & BA & 66 & 86 & 0 & 0 & 0 & 679427 \\
\hline TUP ESTALEIRO ATLÂNTICO SUL & $\mathrm{PE}$ & 67 & 6 & 0 & 1990 & 0 & 0 \\
\hline TUP ESTALEIRO MAUÁ & $\mathrm{RJ}$ & 68 & 4 & 0 & 10799 & 0 & 0 \\
\hline TUP FIBRIA & $\mathrm{BA}$ & 69 & 453 & 0 & 2179533 & 0 & 0 \\
\hline TUP FOGAS & $\mathrm{RO}$ & 70 & 83 & 0 & 0 & 0 & 44693 \\
\hline TUP GERDAU SALVADOR & $\mathrm{BA}$ & 71 & 16 & 0 & 0 & 357979 & 0 \\
\hline TUP GRANEL QUÍMICA & MS & 72 & 1406 & 0 & 1141 & 1800407 & 0 \\
\hline TUP GREGÓRIO CURVO & MS & 73 & 1379 & 0 & 0 & 1913012 & 0 \\
\hline TUP GUAMARÉ & $\mathrm{RN}$ & 74 & 69 & 0 & 0 & 0 & 2848890 \\
\hline TUP HERMASA GRANELEIRO & $\mathrm{AM}$ & 75 & 1485 & 0 & 0 & 4947193 & 130987 \\
\hline TUP IBEPAR MANAUS & $\mathrm{AM}$ & 76 & 699 & 0 & 810802 & 0 & 0 \\
\hline TUP ICOLUB & RJ & 77 & 18 & 0 & 0 & 0 & 58313 \\
\hline TUP ILHA DO GOVERNADOR & RJ & 78 & 83 & 0 & 0 & 0 & 277175 \\
\hline TUP ILHA REDONDA & RJ & 79 & 60 & 0 & 0 & 0 & 172935 \\
\hline TUP IPIRANGA BASE DE & & & & & & & \\
\hline PORTO VELHO & $\mathrm{RO}$ & 80 & 159 & 0 & 0 & 0 & 188777 \\
\hline TUP J. F. OLIVEIRA MANAUS & $\mathrm{AM}$ & 81 & 1265 & 0 & 475140 & 0 & 0 \\
\hline TUP MADRE DE DEUS & BA & 82 & 596 & 0 & 0 & 0 & 21658011 \\
\hline TUP MANAUS & $\mathrm{AM}$ & 83 & 1044 & 0 & 0 & 12489 & 6613384 \\
\hline TUP MARITIMO DE BELMONTE & $\mathrm{BA}$ & 84 & 159 & 0 & 1119160 & 0 & 0 \\
\hline TUP MITA & RS & 85 & 208 & 0 & 0 & 328004 & 0 \\
\hline TUP MOSS & $\mathrm{AM}$ & 86 & 1573 & 0 & 173766 & 0 & 0 \\
\hline TUP MUNGUBA & PA & 87 & 33 & 0 & 279915 & 0 & 15425 \\
\hline TUP NAVECUNHA & $\mathrm{AM}$ & 88 & 495 & 0 & 0 & 0 & 4856 \\
\hline TUP NITERÓI & RS & 89 & 100 & 0 & 0 & 0 & 299072 \\
\hline TUP NORTE CAPIXABA & ES & 90 & 37 & 0 & 0 & 0 & 913033 \\
\hline
\end{tabular}


Tabela 1. Outputs observados para cada unidade produtiva (porto/TUP), em 2012

\begin{tabular}{|c|c|c|c|c|c|c|c|}
\hline Porto/TUPs & $\boldsymbol{U} \boldsymbol{F}$ & $D M U$ & $\begin{array}{l}\text { Núm. de } \\
\text { Atracaçôes }\end{array}$ & Qtde TEUs & $\begin{array}{l}\text { Carga Solta } \\
(t)\end{array}$ & $\begin{array}{l}\text { Granel } \\
\text { Sólido }(t)\end{array}$ & $\begin{array}{l}\text { Granel } \\
\text { Líquido(t) }\end{array}$ \\
\hline TUP OCRIM & AM & 91 & 6 & 0 & 0 & 69301 & 0 \\
\hline TUP OLEOPLAN & RS & 92 & 102 & 0 & 0 & 347994 & 2509 \\
\hline TUP OMNIA & PA & 93 & 86 & 0 & 0 & 4352467 & 0 \\
\hline TUP PASSARÃO & RO & 94 & 757 & 0 & 286721 & 0 & 0 \\
\hline TUP PECÉM & $\mathrm{CE}$ & 95 & 955 & 149103 & 836413 & 1406512 & 521072 \\
\hline TUP PONTA DA LAJE & BA & 96 & 78 & 0 & 156331 & & 0 \\
\hline TUP PONTA DA MADEIRA & MA & 97 & 533 & 0 & 0 & 105033621 & 0 \\
\hline TUP PONTA DA MONTANHA & PA & 98 & 19 & 0 & 20014 & 29974 & 280309 \\
\hline TUP PONTA DE UBU & ES & 99 & 370 & 0 & 32829 & 23385128 & 94632 \\
\hline TUP PORTO CRAI & PA & 100 & 74 & 0 & 0 & 0 & 71903 \\
\hline TUP PORTO ITAPOÁ & $\mathrm{SC}$ & 101 & 425 & 270415 & 0 & 0 & 0 \\
\hline TUP PORTO MURUCUPI & PA & 102 & 79 & 0 & 1 & 806787 & 653669 \\
\hline TUP PORTO SOBRAMIL & MS & 103 & 475 & 0 & 0 & 648173 & 0 \\
\hline TUP PORTO TROMBETAS & PA & 104 & 329 & 0 & 0 & 16391516 & 0 \\
\hline TUP PORTOCEL & ES & 105 & 846 & 0 & 8902095 & 125805 & 0 \\
\hline TUP PORTONAVE & $\mathrm{SC}$ & 106 & 581 & 618434 & 0 & 0 & 0 \\
\hline TUP PRAIA MOLE & ES & 107 & 436 & 0 & 5200434 & 267871 & 0 \\
\hline TUP RIO DOS SINOS & $\mathrm{RS}$ & 108 & 259 & 0 & 0 & 502173 & 69846 \\
\hline TUP SANAVE & AM & 109 & 60 & 0 & 18166 & 7019 & 50 \\
\hline TUP SANTA CLARA & RS & 110 & 222 & 0 & 581233 & 0 & 16547 \\
\hline TUP SÃO FRANCISCO DO SUL & $\mathrm{SC}$ & 111 & 111 & 0 & 0 & 0 & 10274072 \\
\hline TUP SHV & $\mathrm{RS}$ & 112 & 52 & 0 & 0 & 0 & 46616 \\
\hline TUP SOLIMÕES & AM & 113 & 484 & 0 & 0 & 4009 & 2088133 \\
\hline TUP SUCOCÍTRICO CUTRALE & $\mathrm{SP}$ & 114 & 52 & 0 & 0 & 577573 & 675118 \\
\hline TUP SUPER TERMINAIS & AM & 115 & 137 & 187423 & 0 & 0 & 0 \\
\hline TUP T.M. BARCAÇAS OCEÂNICAS & ES & 116 & 59 & 0 & 553397 & 0 & 0 \\
\hline TUP TEPORTI & $\mathrm{SC}$ & 117 & 47 & 19 & 183522 & 0 & 0 \\
\hline TUP TERGASUL & $\mathrm{RS}$ & 118 & 65 & 0 & 0 & 0 & 80008 \\
\hline TUP TERMINAL DA ILHA GUAÍBA & RJ & 119 & 200 & 0 & 0 & 37469523 & 0 \\
\hline TUP TERMINAL DE MINÉRIOS E & & & & & & & \\
\hline METÁLICOS AMAPÁ & $\mathrm{AP}$ & 120 & 144 & 0 & 0 & 6463087 & 0 \\
\hline TUP TERMINAL MARÍTIMO BRAS- & & & & & & & \\
\hline KEM & $\mathrm{RJ}$ & 121 & 36 & 0 & 0 & 0 & 105468 \\
\hline TUP TERMINAL MARÍTIMO INÁCIO & & & & & & & \\
\hline BARBOSA & SE & 122 & 311 & 0 & 878716 & 958722 & 86 \\
\hline TUP TERMINAL MARÍTIMO LUIZ & & & & & & & \\
\hline FOGLIATTO & RS & 123 & 118 & 0 & 317 & 1632097 & 0 \\
\hline TUP TERMINAL PORTUÁRIO & & & & & & & \\
\hline TKCSA & $\mathrm{RJ}$ & 124 & 109 & 0 & 3424120 & 3427848 & 0 \\
\hline TUP TRANSPORTES CARINHOSO & AM & 125 & 307 & 0 & 180887 & 0 & 0 \\
\hline TUP ULTRAFÉRTIL & $\mathrm{SP}$ & 126 & 108 & 0 & 0 & 2244128 & 378147 \\
\hline TUP USIMINAS & $\mathrm{SP}$ & 127 & 212 & 0 & 1394698 & 4332827 & 0 \\
\hline TUP UTC ENGENHARIA & RJ & 128 & 8 & 0 & 9319 & 0 & 0 \\
\hline TUP VILA VELHA & ES & 129 & 1081 & 0 & 107568 & 26558 & 195463 \\
\hline TUP WELLSTREAM & $\mathrm{RJ}$ & 130 & 43 & 0 & 36080 & 0 & 0 \\
\hline TUP YARA BRASIL FERTILIZANTES & RS & 131 & 284 & 0 & 0 & 2054392 & 148979 \\
\hline VILA DO CONDE & PA & 132 & 742 & 38879 & 976467 & 11814287 & 2021929 \\
\hline VITÓRIA & ES & 133 & 1286 & 270408 & 1164674 & 1752598 & 745844 \\
\hline
\end{tabular}

Com tal estrutura de variáveis, busca-se analisar o setor portuário, estritamente no que tange à movimentação de carga, avaliando-se cada unidade, em termos de relevância comercial, sem levar em conta os aspectos logísticos intrínsecos à atividade. Isso porque este trabalho avalia a produção dos portos, em termos de carga movimentada e quantidade de atracações. Trata-se de uma perspectiva diferente daquela comumente utilizada na literatura, mas que permite uma análise voltada para a atividade-fim portuária. Assim, um índice inferior a $100 \%$ pode ser motivado tanto pela má gestão de recursos quanto pela deficiência em algum aspecto não considerado (e.g., infraestrutura, capital, mão-deobra).

\subsection{Agrupamento das unidades portuárias}

As unidades portuárias foram segregadas, conforme a natureza da carga por elas movimentada, de modo análogo ao proposto em Cook et al. (2013), gerando oito grupos (clusters) disjuntos. A Tabela 2 apresenta a divisão das unidades em clusters, segundo a natureza da carga operada.

Ressalte-se que as unidades de cada grupo operam somente com o(s) tipo(s) de carga identificado(s) para cada cluster na Tabela 2, ou seja, as unidades do cluster 1, por exemplo, operaram somente com carga conteinerizada, não tendo movimentado outro tipo de carga durante o período considerado neste estudo. Assim, dos outputs definidos na subseção anterior, são considerados na avaliação das unidades de cada cluster somente aqueles referentes ao tipo de 
RUBEM, A.P.S.; BRANDÃO, L.C.; SOARES DE MELLO, J.C.C.B.

Tabela 2. Composição dos clusters, conforme o tipo de carga operado pelas unidades

\begin{tabular}{lc}
\hline Cluster & Composição \\
\hline 1 & Carga conteinerizada \\
2 & Carga solta \\
3 & Carga em granel sólido \\
4 & Carga em granel líquido \\
5 & Todos os tipos de carga \\
6 & Carga solta e em granel sólido \\
7 & Carga solta, em granel sólido e granel líquido \\
8 & Carga em granel sólido e granel líquido \\
\hline
\end{tabular}

Tabela 3. Resultados do cluster 1 (unidades que só operam contêineres)

\begin{tabular}{|c|c|c|c|c|c|c|}
\hline \multirow{2}{*}{ Porto/TUP } & \multirow{2}{*}{$\boldsymbol{U} \boldsymbol{F}$} & \multicolumn{2}{|c|}{ Índice DEA } & \multicolumn{3}{|c|}{ Ranking } \\
\hline & & Padrão & Composto & Padrão & Composto & Copeland \\
\hline TUP PORTONAVE & $\mathrm{SC}$ & $100 \%$ & $100 \%$ & 1 & 1 & 1 \\
\hline ITAJAÍ & $\mathrm{SC}$ & $63 \%$ & $67 \%$ & 3 & 2 & 2 \\
\hline TUP PORTO ITAPOÁ & $\mathrm{SC}$ & $73 \%$ & $61 \%$ & 2 & 3 & 3 \\
\hline TUP CHIBATÃO & $\mathrm{AM}$ & $44 \%$ & $38 \%$ & 4 & 4 & 4 \\
\hline TUP SUPER TERMINAIS & $\mathrm{AM}$ & $30 \%$ & $18 \%$ & 5 & 5 & 5 \\
\hline
\end{tabular}

carga efetivamente movimentado pelas unidades nele inseridas, além do número de atracações, este último output comum a todos os clusters. Desse modo, por exemplo, para o cluster 1, os outputs são apenas movimentação de carga conteinerizada (em TEUs) e número de atracações.

Considerando todas as combinações de tipo de carga possíveis, deveria haver treze clusters. No entanto, em 2012, nenhuma unidade operou conjuntamente contêineres e granel sólido, e tampouco contêineres e granel líquido. Além disso, apenas uma unidade operou conjuntamente contêineres e carga solta; duas unidades operaram conjuntamente contêineres carga solta e em granel sólido; e duas unidades operaram conjuntamente com carga solta e em granel líquido. Entretanto, o número de unidades em cada cluster deve ser no mínimo três vezes o número de inputs e outputs do modelo (ou ao menos se aproximar desta proporção), para que os resultados DEA sejam confiáveis (Banker et al., 1989). Assim, essas cinco unidades que estariam nos três grupos supracitados foram retiradas da análise, restando 128 unidades, divididas nos oito clusters da Tabela 2.

\subsection{Aplicação dos Métodos}

Iniciou-se com a abordagem DEA (as DMUs são as 128 unidades portuárias), aplicando-se o modelo em (1), separadamente para cada cluster (vide Tabela 2), e considerando-se os outputs a eles afetos (i.e., os valores não-nulos apresentados na Tabela 1 para cada DMU). Alguns clusters contêm menos DMUs do que a proporção sugerida em Banker et al. (1989), indicada na subseção anterior, o que poderia acarretar baixa discriminação. Portanto, ao invés do índice padrão, optou-se pelo índice composto normalizado (Soares de Mello et al., 2008), dado pela média entre o índice da fronteira padrão e o complemento do índice resultante da análise da fronteira invertida (Yamada et al., 1994; Entani et al., 2002), que considera os inputs originais como outputs, e vice-versa.

Em seguida, aplicou-se o método de Copeland às unidades de cada cluster. Enquanto DEA analisa o valor das variáveis de cada DMU, Copeland avalia apenas a ordenação. Por outro lado, DEA só considera a distância até a fronteira, ao passo que Copeland analisa a posição relativa de cada alternativa (DMU) perante todas as outras. Nesse sentido, o índice composto normalizado representa uma alternativa intermediária, pois avalia a distância até as fronteiras padrão e invertida, além dos valores das variáveis em si.

\section{RESULTADOS}

As Tabelas 3 a 10 exibem os resultados das duas abordagens utilizadas, para cada um dos clusters formados, em termos de índices e ordenações, os quais foram obtidos mediante o emprego dos softwares SIAD (Angulo-Meza et al., 2005) e WebPROA (www.webproa.com). As unidades portuárias estão elencadas nas tabelas a seguir, obedecendo a ordenação do índice composto normalizado, explicado na subseção anterior, a qual daqui por diante será chamada de ordenação composta, por simplicidade.

Na Tabela 3, referente ao cluster 1 , nota-se que quatro das cinco unidades especializadas em contêineres são TUPs, e que todas as unidades se restringem a Santa Catarina e Amazonas. Enquanto os melhores colocados (marítimas) estão no sul, ao norte estão aqueles (fluviais) com os piores resultados. Trata-se de um cluster relativamente homogêneo (índice médio padrão de $62 \%$ ). Além disso, as ordenações são parecidas, sendo a composta idêntica à de Copeland. Tal equivalência se deve às poucas unidades e à homogeneidade: a comparação entre as fronteiras (padrão e invertida) usada pelo índice composto se aproxima da comparação entre os pares de alternativas (Copeland).

$\mathrm{Na}$ Tabela 4, relativa ao cluster 2 , nota-se que 22 das 23 unidades especializadas em carga solta são TUPs. Na verdade, o único porto (Niterói) foi inserido neste cluster, pois ainda não operava granel líquido de forma regular ( $98 t$, em abril/2010; e 64t, em outubro/2012). A regularidade se dá a partir de 2013. Logo, atualmente, somente TUPs são especializados em carga solta.

Observa-se que as ordenações DEA padrão e composta da Tabela 4 resultaram próximas, sugerindo um cluster de unidades generalistas: aquelas com um nível elevado de movimentação de carga solta também tiveram um número alto de atracações, indicando unidades com calado semelhante. A ordenação Copeland também foi similar, exceto para os TUPs Bertolini Belém e Moss. 
Tabela 4. Resultados do cluster 2 (unidades que só operam carga solta)

\begin{tabular}{|c|c|c|c|c|c|c|}
\hline \multirow{2}{*}{ Porto/TUP } & \multirow{2}{*}{$\boldsymbol{U} \boldsymbol{F}$} & \multicolumn{2}{|c|}{ Índice DEA } & \multicolumn{3}{|c|}{ Ranking } \\
\hline & & Padrão & Composto & Padrão & Composto & Copeland \\
\hline TUP CHIBATÃO 2 & AM & $100 \%$ & $100 \%$ & 1 & 1 & $\overline{1}$ \\
\hline TUP FIBRIA & $\mathrm{BA}$ & $100 \%$ & $100 \%$ & 1 & 2 & 3 \\
\hline TUP MOSS & $\mathrm{AM}$ & $100 \%$ & $100 \%$ & 1 & 3 & 6 \\
\hline TUP J. F. OLIVEIRA MANAUS & $\mathrm{AM}$ & $83 \%$ & $92 \%$ & 4 & 4 & 4 \\
\hline TUP BERTOLINI BELÉM & $\mathrm{PA}$ & $79 \%$ & $89 \%$ & 5 & 5 & 2 \\
\hline TUP IBEPAR MANAUS & $\mathrm{AM}$ & $62 \%$ & $81 \%$ & 6 & 6 & 5 \\
\hline TUP PASSARÃO & RO & $50 \%$ & $75 \%$ & 8 & 7 & 7 \\
\hline TUP MARITIMO DE BELMONTE & $\mathrm{BA}$ & $51 \%$ & $75 \%$ & 7 & 8 & 7 \\
\hline TUP BERTOLINI SANTARÉM & $\mathrm{PA}$ & $47 \%$ & $73 \%$ & 9 & 9 & 10 \\
\hline NITERÓI & RJ & $25 \%$ & $61 \%$ & 11 & 10 & 13 \\
\hline TUP TRANSPORTE CARINHOSO & $\mathrm{AM}$ & $21 \%$ & $60 \%$ & 12 & 11 & 9 \\
\hline TUP T.M. BARCAÇAS OCEÂNICAS & ES & $25 \%$ & $59 \%$ & 10 & 12 & 12 \\
\hline TUP CMPC GUAIIBA & RS & $18 \%$ & $58 \%$ & 13 & 13 & 11 \\
\hline TUP BELMONTE & RO & $13 \%$ & $55 \%$ & 14 & 14 & 13 \\
\hline TUP BERTOLINI SANTANA & $\mathrm{AP}$ & $12 \%$ & $55 \%$ & 15 & 15 & 13 \\
\hline TUP PONTA DA LAJE & BA & $9 \%$ & $52 \%$ & 16 & 16 & 16 \\
\hline TUP CAIMA & RO & $7 \%$ & $51 \%$ & 18 & 17 & 18 \\
\hline TUP BRASKARNE & $\mathrm{SC}$ & $8 \%$ & $46 \%$ & 17 & 18 & 17 \\
\hline TUP WELLSTREAM & RJ & $3 \%$ & $45 \%$ & 19 & 19 & 19 \\
\hline TUP BRASFELS & RJ & $1.2 \%$ & $29 \%$ & 20 & 20 & 20 \\
\hline TUP UTC ENGENHARIA & RJ & $0.7 \%$ & $18 \%$ & 21 & 21 & 21 \\
\hline TUP ESTALEIRO MAUÁ & RJ & $0.6 \%$ & $0.3 \%$ & 22 & 22 & 22 \\
\hline TUP ESTALEIRO ATLÂNTICO SUL & $\mathrm{PE}$ & $0.4 \%$ & $0.2 \%$ & 23 & 23 & 23 \\
\hline
\end{tabular}

Tabela 5. Resultados do cluster 3 (unidades que só operam granel sólido)

\begin{tabular}{|c|c|c|c|c|c|c|}
\hline \multirow{2}{*}{ Porto/TUP } & \multirow{2}{*}{$\boldsymbol{U} \boldsymbol{F}$} & \multicolumn{2}{|c|}{ Índice $D E A$} & \multicolumn{3}{|c|}{ Ranking } \\
\hline & & Padrão & Composto & Padrão & Composto & Copeland \\
\hline TUP GREGÓRIO CURVO & MS & $100 \%$ & $100 \%$ & 1 & 1 & 3 \\
\hline TUP COPELMI & RS & $100 \%$ & $99 \%$ & 1 & 3 & 14 \\
\hline TUP PORTO SOBRAMIL & MS & $75 \%$ & $87 \%$ & 4 & 4 & 8 \\
\hline AREIA BRANCA & $\mathrm{RN}$ & $75 \%$ & $87 \%$ & 5 & 5 & 3 \\
\hline TUP TERMINAL DA ILHA GUAÍBA & RJ & $36 \%$ & $68 \%$ & 7 & 7 & 5 \\
\hline TUP PORTO TROMBETAS & PA & $33 \%$ & $66 \%$ & 8 & 8 & 2 \\
\hline TUP CVRD PRAIA MOLE & $\mathrm{ES}$ & $18 \%$ & $58 \%$ & 9 & 9 & 7 \\
\hline TUP MITA & RS & $15 \%$ & $57 \%$ & 10 & 10 & 11 \\
\hline TUP CIMBAGÉ & RS & $14 \%$ & $56 \%$ & 12 & 11 & 13 \\
\hline TUP CIMENTO VENCEMOS & AM & $2 \%$ & $45 \%$ & 16 & 15 & 16 \\
\hline TUP GERDAU SALVADOR & BA & $1 \%$ & $41 \%$ & 17 & 16 & 15 \\
\hline TUP OCRIM & AM & $0.5 \%$ & $25 \%$ & 19 & 17 & 17 \\
\hline PELOTAS & $\mathrm{RS}$ & $0.4 \%$ & $25 \%$ & 20 & 18 & 18 \\
\hline ETC ITACAL & $\mathrm{AM}$ & $7 \%$ & $3 \%$ & 15 & 19 & 19 \\
\hline ETC PORTO MURTINHO & MS & $1 \%$ & $0.5 \%$ & 18 & 20 & 21 \\
\hline ESTRELA & RS & $0.2 \%$ & $0.1 \%$ & 21 & 21 & 20 \\
\hline
\end{tabular}

Na Tabela 5, relativa ao cluster 3 , nota-se que 16 das 21 unidades especializadas em granel sólido são TUPs, mantendo-se um equilíbrio razoável com a proporção geral. As ordenações DEA padrão e composta são bem similares, indicando unidades generalistas e, consequentemente, unidades com calado máximo semelhante.

Em alguns casos da Tabela 5, a ordenação em Copeland se afastou das demais ordenações, mas pareceu ser mais justa, pelas razões a seguir. O TUP Copelmi teve um número mediano de atracações e valor de carga movimentada bem inferior ao resto do grupo. Portanto, o 14을 lugar parece mais adequado. O TUP Porto Trombetas apresentou número mediano de atracações e elevada movimentação de carga. Para essa unidade, o 2o lugar também parece mais apropriado. O TUP Ponta da Madeira (1ํ lugar, em quaisquer dos métodos) movimentou o maior volume de carga, mas teve um nível mediano de atracações, indicando um calado maior. O Porto de Estrela (pior colocado em DEA e penúltimo no Copeland) teve o pior nível de atracações (igual ao ETC Porto Murtinho) e baixa movimentação de carga.

A Tabela 6, relativa ao cluster 4, mostra que as 24 unidades especializadas em granel líquido são TUPs. Isso sugere que esse tipo de carga exige menos infraestrutura, podendo ser operado por unidades mais enxutas. Para o TUP Navecunha, as ordenações DEA composta e Copeland são similares, embora resultem em colocação pior que a padrão. Isso porque a unidade teve um bom nível de atracação, 
RUBEM, A.P.S.; BRANDÃO, L.C.; SOARES DE MELLO, J.C.C.B.

Tabela 6. Resultados do cluster 4 (unidades que só operam granel líquido)

\begin{tabular}{|c|c|c|c|c|c|c|}
\hline \multirow{2}{*}{ Porto/TUP } & \multirow{2}{*}{$\boldsymbol{U} \boldsymbol{F}$} & \multicolumn{2}{|c|}{ Índice $D E A$} & \multirow{2}{*}{$\begin{array}{l}\text { Ranking } \\
\text { Padrão }\end{array}$} & \multirow[b]{2}{*}{ Composto } & \multirow[b]{2}{*}{ Copeland } \\
\hline & & Padrão & Composto & & & \\
\hline TUP ALMIRANTE BARROSO & SP & $100 \%$ & $100 \%$ & 1 & 1 & 1 \\
\hline TUP MADRE DE DEUS & BA & $97 \%$ & $98 \%$ & 2 & 2 & 2 \\
\hline TUP ALMIRANTE SOARES DUTRA & $\mathrm{RS}$ & $31 \%$ & $62 \%$ & 4 & 3 & 3 \\
\hline TUP CATTALINI & PR & $24 \%$ & $57 \%$ & 6 & 4 & 5 \\
\hline TUP SÃO FRANCISCO DO SUL & $\mathrm{SC}$ & $20 \%$ & $53 \%$ & 8 & 5 & 4 \\
\hline TUP DOW BRASIL GUARUJÁ & SP & $17 \%$ & $51 \%$ & 9 & 6 & 7 \\
\hline TUP IPIRANGA BASE D PORTO VELHO & RO & $26 \%$ & $50 \%$ & 5 & 7 & 8 \\
\hline TUP CARMÓPOLIS & $\mathrm{SE}$ & $17 \%$ & $50 \%$ & 10 & 8 & 6 \\
\hline TUP NITERÓI & $\mathrm{RS}$ & $16 \%$ & $49 \%$ & 11 & 9 & 12 \\
\hline TUP AGROPALMA & PA & $22 \%$ & $49 \%$ & 7 & 10 & 9 \\
\hline TUP DOW ARATU & $\mathrm{BA}$ & $14 \%$ & $47 \%$ & 12 & 11 & 12 \\
\hline TUP BRASKEM ALAGOAS & $\mathrm{AL}$ & $14 \%$ & $47 \%$ & 13 & 12 & 9 \\
\hline TUP ILHA DO GOVERNADOR & RJ & $13 \%$ & $47 \%$ & 14 & 13 & 15 \\
\hline TUP GUAMARÉ & $\mathrm{RN}$ & $11 \%$ & $43 \%$ & 17 & 14 & 9 \\
\hline TUP NAVECUNHA & $\mathrm{AM}$ & $80 \%$ & $41 \%$ & 3 & 15 & 14 \\
\hline TUP ILHA REDONDA & RJ & $10 \%$ & $39 \%$ & 19 & 16 & 18 \\
\hline TUP DE GNL DA BAÍA DA GUANABARA & RJ & $7 \%$ & $35 \%$ & 21 & 17 & 15 \\
\hline TUP NORTE CAPIXABA & ES & $6 \%$ & $29 \%$ & 22 & 18 & 17 \\
\hline TUP TERMINAL MARÍTIMO BRASKEM & RJ & $6 \%$ & $26 \%$ & 23 & 19 & 22 \\
\hline TUP TERGASUL & RS & $11 \%$ & $24 \%$ & 18 & 20 & 20 \\
\hline TUP PORTO CRAI & PA & $12 \%$ & $23 \%$ & 16 & 21 & 18 \\
\hline TUP FOGAS & $\mathrm{RO}$ & $13 \%$ & $8 \%$ & 14 & 22 & 21 \\
\hline TUP SHV & $\mathrm{RS}$ & $8 \%$ & $4 \%$ & 20 & 23 & 23 \\
\hline TUP ICOLUB & RJ & $3 \%$ & $1 \%$ & 24 & 24 & 24 \\
\hline
\end{tabular}

Tabela 7. Resultados do cluster 5 (unidades que operam todos os tipos de carga)

\begin{tabular}{llllccc}
\hline \multirow{2}{*}{ Porto/TUP } & \multirow{2}{*}{$\boldsymbol{U}$} & \multicolumn{1}{c}{ Indice $\boldsymbol{D E} \boldsymbol{A}$} & Ranking & & \\
\cline { 3 - 7 } & & Padrão & Composto & Padrão & Composto & Copeland \\
\hline SANTOS & $100 \%$ & $100 \%$ & 1 & 1 & 1 \\
PARANAGUÁ & PR & $68 \%$ & $82 \%$ & 3 & 2 & 2 \\
SÃO FRANCISCO DO SUL & SC & $73 \%$ & $77 \%$ & 2 & 3 & 8 \\
RIO GRANDE & RS & $45 \%$ & $71 \%$ & 6 & 4 & 3 \\
VITÓRIA & ES & $35 \%$ & $63 \%$ & 7 & 5 & 4 \\
SUAPE & PE & $46 \%$ & $61 \%$ & 5 & 6 & 5 \\
RIO DE JANEIRO & RJ & $26 \%$ & $58 \%$ & 11 & 7 & 8 \\
TUP PECÉM & CE & $25 \%$ & $54 \%$ & 12 & 8 & 7 \\
VILA DO CONDE & PA & $29 \%$ & $48 \%$ & 10 & 9 & 5 \\
FORTALEZA & CE & $20 \%$ & $42 \%$ & 14 & 10 & 12 \\
ITAQUI & MA & $62 \%$ & $39 \%$ & 4 & 11 & 10 \\
SALVADOR & BA & $10 \%$ & $31 \%$ & 15 & 12 & 15 \\
PORTO VELHO & RO & $35 \%$ & $18 \%$ & 8 & 13 & 11 \\
SANTARÉM & PA & $30 \%$ & $15 \%$ & 9 & 14 & 12 \\
BELÉM & PA & $22 \%$ & $15 \%$ & 13 & 15 & 12 \\
IMBITUBA & SC & $4 \%$ & $5 \%$ & 17 & 16 & 16 \\
RECIFE & PE & $7 \%$ & $3 \%$ & 16 & 17 & 17 \\
NATAL & RN & $2 \%$ & $1 \%$ & 18 & 18 & 18 \\
\hline
\end{tabular}

mas um volume relativamente pequeno de carga movimentada. Trata-se, portanto, de uma unidade com baixo calado máximo. Nesse caso, as ordenações DEA composta e Copeland parecem mais adequadas. O TUP Almirante Barroso (1- lugar, em todos os métodos) teve os maiores níveis de atracações e carga movimentada, enquanto o TUP Icolub (pior colocado, em qualquer método) teve o menor número de atracações.

A Tabela 7 , relativa ao cluster 5 , mostra que 17 das 18 unidades que operam carga de todo tipo são portos, denotando que tal diversificação requer estrutura robusta. Diferentemente da ordenação DEA padrão, as ordenações composta e de Copeland são similares. Isso significa que há portos especialistas, que são bem avaliados com o índice padrão, mas não com o composto. Esse é o caso do porto de Itaqui com boa movimentação de granéis sólido e líquido, mas nem tanto em termos de contêineres e carga solta. Situação semelhante ocorre com os portos de Porto Velho e Santarém. Ambos tiveram baixa movimentação de contêineres e mediana de granel sólido, sendo o primeiro fraco em granel líquido e o segundo em carga solta. A ordenação DEA padrão os considerou medianos, e as demais consideraram-nos fracos. O porto de Santos (1ํo lugar, em quaisquer dos métodos) praticou os maiores níveis em todos os outputs. O porto de Natal (pior colocado em todos os métodos) teve os piores resultados de atracação e movimentação de carga solta e em granéis sólido e líquido.

No cluster 6 , apresentado na Tabela 8 , foram incluídos o TUP Sanave e o porto do Forno, que embora tenham operado outros tipos de carga, não o fizeram de forma regular. O primeiro movimentou $50 t$ de granel líquido em junho; e o segundo um total de 6 TEUs, em fevereiro e março. Nos 
Tabela 8. Resultados do cluster 6 (unidades que só operam carga solta e granel sólido)

\begin{tabular}{|c|c|c|c|c|c|c|}
\hline \multirow{2}{*}{ Porto/TUP } & \multirow{2}{*}{$\boldsymbol{U} \boldsymbol{F}$} & \multicolumn{2}{|c|}{ Índice DEA } & \multicolumn{3}{|l|}{ Ranking } \\
\hline & & Padrão & Composto & Padrão & Composto & Copeland \\
\hline TUP PORTOCEL & $\mathrm{ES}$ & $100 \%$ & $100 \%$ & 1 & 1 & 1 \\
\hline TUP USIMINAS & SP & $100 \%$ & $99 \%$ & 1 & 2 & 2 \\
\hline TUP TERMINAL PORTUÁRIO TKCSA & RJ & $100 \%$ & $94 \%$ & 1 & 3 & 5 \\
\hline TUP PRAIA MOLE & ES & $62 \%$ & $78 \%$ & 6 & 4 & 2 \\
\hline TUP GRANEL QUÍMICA & MS & $100 \%$ & $61 \%$ & 1 & 5 & 2 \\
\hline ANTONINA & PR & $28 \%$ & $55 \%$ & 8 & 6 & 6 \\
\hline TUP SANAVE & $\mathrm{AM}$ & $100 \%$ & $52 \%$ & 1 & 7 & 10 \\
\hline ILHÉUS & $\mathrm{BA}$ & $10 \%$ & $35 \%$ & 10 & 8 & 9 \\
\hline PORTO ALEGRE & $\mathrm{RS}$ & $27 \%$ & $29 \%$ & 9 & 9 & 6 \\
\hline $\begin{array}{l}\text { TUP TERMINAL MARÍTIMO LUIZ FO- } \\
\text { GLIATTO }\end{array}$ & $\mathrm{RS}$ & $39 \%$ & $20 \%$ & 7 & 10 & 6 \\
\hline FORNO & RJ & $4 \%$ & $2 \%$ & 11 & 11 & 11 \\
\hline
\end{tabular}

dois casos, não há outro registro do tipo desde 2010. As ordenações DEA destoam entre si, devido à baixa discriminação do índice padrão (5 unidades na fronteira). Aqui, o índice composto cumpriu seu papel de aumentar a discriminação em DEA.

O TUP Usiminas é considerado inferior ao TUP Portocel nas ordenações composta e Copeland, apesar do bom nível de carga solta e do maior nível de granel sólido do cluster, porque essas ordenações dão preferência a unidades generalistas, diferentemente de DEA padrão. O TUP Portocel (1º lugar em todos os métodos) operou um volume altíssimo de carga solta, um bom nível de atracações e um volume baixo de granel sólido. O porto do Forno (pior colocado em todos os métodos) teve o menor nível de atracações, além de um nível baixo de cargas solta e granel sólido.
A Tabela 9, afeta ao cluster 7 , mostra que 5 das 9 unidades especializadas em carga solta e granéis sólidos e líquidos são TUPs. Neste cluster, as ordenações DEA composta e Copeland claramente dão preferência às unidades generalistas. O TUP Vila Velha exibe o maior nível de atracações (quase o triplo do $2^{\circ}$ maior), mas obteve $5^{\circ}$ lugar na composta e $2^{\circ}$ em Copeland. O TUP Terminal Marítimo Inácio Barbosa teve a maior movimentação de carga solta ( 8 vezes a $2^{\text {a }}$ maior), mas ficou em $5^{\circ}$ lugar nas ordenações composta e Copeland. O TUP Ponta de Ubu teve a maior movimentação de granel sólido (12 vezes a $2^{-}$- maior), mas obteve $4^{\circ}$ lugar na ordenação composta e $2^{\circ}$ lugar em Copeland. O Porto de Maceió (1ํo lugar em qualquer método) teve 2- maior movimentação de granel sólido e maior de granel líquido. O TUP Caulim da Amazônia (pior colocado em

Tabela 9. Resultados do cluster 7 (unidades que operam carga solta, granel sólido e líquido)

\begin{tabular}{|c|c|c|c|c|c|c|}
\hline \multirow{2}{*}{ Porto/TUP } & \multirow{2}{*}{$\boldsymbol{U F}$} & \multicolumn{2}{|c|}{ Índice DEA } & \multicolumn{3}{|c|}{ Ranking } \\
\hline & & Padrão & Composto & Padrão & Composto & Copeland \\
\hline MACEIÓ & $\mathrm{AL}$ & $100 \%$ & $100 \%$ & 1 & 1 & 1 \\
\hline CABEDELO & PB & $98 \%$ & $92 \%$ & 6 & 2 & 2 \\
\hline MACAPÁ & AP & $100 \%$ & $85 \%$ & 1 & 3 & 5 \\
\hline TUP PONTA DE UBU & ES & $100 \%$ & $78 \%$ & 1 & 4 & 2 \\
\hline TUP TERMINAL MARÍTIMO INÁCIO BARBOSA & SE & $100 \%$ & $54.8 \%$ & 1 & 5 & 5 \\
\hline TUP VILA VELHA & ES & $100 \%$ & $54.8 \%$ & 1 & 5 & 2 \\
\hline TUP PONTA DA MONTANHA & PA & $31 \%$ & $17 \%$ & 7 & 7 & 8 \\
\hline ANGRA DOS REIS & RJ & $11 \%$ & $6 \%$ & 8 & 8 & 7 \\
\hline TUP CAULIM DA AMAZÔNIA & PA & $7 \%$ & $4 \%$ & 9 & 9 & 8 \\
\hline
\end{tabular}

Tabela 10. Resultados do cluster 8 (unidades que só operam granéis sólido e líquido)

\begin{tabular}{|c|c|c|c|c|c|c|}
\hline \multirow{2}{*}{ Porto/TUP } & \multirow{2}{*}{$\boldsymbol{U} \boldsymbol{F}$} & \multicolumn{2}{|c|}{ Índice DEA } & \multicolumn{3}{|c|}{ Ranking } \\
\hline & & Padrão & Composto & Padrão & Composto & Copeland \\
\hline TUP ALMIRANTE TAMANDARÉ (ILHA D’ÁGUA) & $\overline{\mathrm{RJ}}$ & $100 \%$ & $100 \%$ & 1 & 1 & 1 \\
\hline TUP CVRD TUBARÃO & ES & $100 \%$ & $99 \%$ & 1 & 2 & 2 \\
\hline TUP HERMASA GRANELEIRO & $\mathrm{AM}$ & $100 \%$ & $98 \%$ & 1 & 3 & 2 \\
\hline TUP ALMIRANTE MAXIMIANO DA FONSECA & RJ & $100 \%$ & $95 \%$ & 1 & 4 & 9 \\
\hline TUP RIO DOS SINOS & RS & $100 \%$ & $90 \%$ & 1 & 5 & 14 \\
\hline TUP MANAUS & $\mathrm{AM}$ & $75 \%$ & $86 \%$ & 7 & 6 & 4 \\
\hline ARATU & BA & $44 \%$ & $70 \%$ & 8 & 7 & 5 \\
\hline TUP BIANCHINI & RS & $30 \%$ & $62 \%$ & 10 & 8 & 8 \\
\hline TUP SOLIMÕES & AM & $34 \%$ & $62 \%$ & 9 & 9 & 7 \\
\hline TUP ALUMAR & MA & $27 \%$ & $59 \%$ & 11 & 10 & 6 \\
\hline TUP YARA BRASIL FERTILIZANTES & $\mathrm{RS}$ & $20 \%$ & $54 \%$ & 12 & 11 & 10 \\
\hline $\begin{array}{l}\text { TUP DNP - BASE DE DISTRIBUIÇÃO SECUNDÁRIA } \\
\text { DE SANTARÉM }\end{array}$ & PA & $100 \%$ & $51 \%$ & 1 & 12 & 17 \\
\hline TUP ULTRAFÉRTIL & SP & $8 \%$ & $39 \%$ & 13 & 13 & 10 \\
\hline TUP CEVAL & RS & $6 \%$ & $35 \%$ & 15 & 14 & 12 \\
\hline TUP PORTO MURUCUPI & PA & $6 \%$ & $33 \%$ & 16 & 15 & 13 \\
\hline TUP SUCOCÍTRICO CUTRALE & SP & $5 \%$ & $21 \%$ & 17 & 16 & 14 \\
\hline TUP OLEOPLAN & RS & $7 \%$ & $3 \%$ & 14 & 17 & 16 \\
\hline
\end{tabular}


qualquer método) teve o $2^{\circ}$ menor nível de atracações e a pior movimentação de carga solta.

$\mathrm{Na}$ Tabela 10, relativa ao cluster 8 , nota-se que 16 das 17 unidades que operam somente granéis sólidos e líquidos são TUPs. O TUP Porto Murucupi foi incluído neste cluster, pois embora tenha operado com carga solta $(1 t$, em jan/2012), o fato constituiu uma excepcionalidade, não observada em outra ocasião desde 2010. Mais uma vez, as ordenações DEA composta e Copeland deram preferência às unidades generalistas. O TUP Almirante Tamandaré (1ำ lugar geral) exibe um bom nível de atracações (2o lugar), uma movimentação mediana de granel sólido e boa de granel líquido (2o lugar). Ressalta-se que o TUP Almirante Maximiano da Fonseca (1o lugar em granel líquido e com quase o triplo do $2^{\circ}$ - maior) ficou em $9^{\circ}$ lugar no Copeland. Por outro lado, a baixa discriminação do índice DEA padrão gerou discrepâncias, como no TUP Rio dos Sinos e no TUP DNP, cujos outputs não justificam o primeiro lugar obtido.

A abordagem DEA aqui usada não permite uma ordenação geral, que englobe todas as unidades portuárias dos diferentes clusters. Isso porque, como o agrupamento das unidades portuárias resultou em clusters disjuntos (interseção nula), a comparação direta entre DMUs pertencentes a clusters diferentes torna-se inviável, uma vez que a eficiência de uma DMU só pode ser comparada àquelas inseridas no mesmo conjunto de análise. A superação de tal inconveniente, visando a uma avaliação global do setor portuário nacional, requer o emprego de técnicas destinadas à homogeneização das unidades produtivas, tal como feito, e.g., em Bertolotto e Soares de Mello (2011) e Gomes et al. (2012), e foge ao escopo deste estudo.

\section{CONSIDERAÇÕES FINAIS}

Neste estudo, realizou-se uma análise do setor portuário brasileiro, englobando unidades que operam diferentes tipos de carga. As unidades portuárias foram agrupadas em oito clusters distintos, conforme a natureza da carga movimentada. Para identificar a relevância comercial de cada unidade, aplicou-se um modelo DEA com input unitário (Lovell e Pastor, 1999), considerando como outputs o total de cada tipo de carga movimentada, bem como o número de atracações. Desse modo, buscou-se avaliá-las, exclusivamente, quanto à importância relativa de suas operações, sem considerar a logística inerente à atividade portuária. Portanto, para as unidades com fraco desempenho, a justificativa pode ser tanto a má gestão como a deficiência em algum aspecto não considerado (e.g., infraestrutura, capital, mão-de-obra).

Como alguns clusters apresentaram poucas unidades, relativamente à regra de Banker et al. (1989), utilizou-se também o índice composto normalizado (Soares de Mello et al., 2008) para elevar a capacidade de discriminação. As ordenações finais foram comparadas às do método multicritério ordinal de Copeland (1951). Em geral, a ordenação resultante do índice DEA composto se assemelha bastante à obtida com o método de Copeland. Em alguns casos, a ordenação de Copeland parece mais adequada, pois é feita a comparação entre todas as unidades, e não apenas entre as situadas nas fronteiras de eficiência e invertida; em outros, o índice composto parece mais apropriado por considerar os valores das variáveis em si, e não só as ordenações.

Assim, o método de Copeland parece mais adequado em situações em que se deseja diferenciar os diferentes tipos de habilidade (diferentes combinações dos critérios de desempenho) entre as unidades avaliadas; enquanto DEA com input unitário torna-se mais adequado para situações em que os valores das variáveis (critérios de desempenho) variam muito entre as unidades avaliadas, e deseja-se leválos em conta. Dessa forma, a análise aqui conduzida auxilia na compreensão das diferenças entre DEA com input unitário e o método de Copeland, indicando o modelo mais adequado para cada tipo de situação.

Em geral, as unidades melhor avaliadas são as que apresentaram bons níveis de atracação, e não apenas de carga movimentada, indicando que a modelagem aqui adotada pretere as unidades com calado máximo elevado, mas que realizam poucas atracações. Em estudos futuros, pretende-se incorporar a aplicação de técnicas de homogeneização (e.g., Bertoloto e Soares de Mello, 2011; Gomes et al., 2012b), de forma a possibilitar a comparação direta entre unidades portuárias que operam em diferentes ramos (i.e., entre clusters distintos).

\section{AGRADECIMENTOS}

Ao CNPq, pelo apoio financeiro.

\section{REFERÊNCIAS}

Acosta, C.M.M.; Silva, A.M.V.A. e Lima, M.L.P.D. (2011) Aplicação de análise envoltória de dados (DEA) para medir eficiência em portos brasileiros. Journal of Transport Literature, v. 5 , n. 4 , p. $88-102$.

Angulo-Meza, L.; Biondi Neto, L.; Soares de Mello, J.C.C.B. e Gomes, E.G. (2005) ISYDS - Integrated System for Decision Support (SIAD - Sistema Integrado de Apoio a Decisão): A software package for data envelopment analysis model. Pesquisa Operacional, v. 25, n. 3, p. 493-503.

DOI: $10.5220 / 0002548802070212$

Arrow, K.J. (1951) Social Choice and Individual Values. New York: Wiley.

Banker, R.D.; Charnes, A.; Cooper, W.W. (1984) Some Models for Estimating Technical and Scale Inefficiencies in Data Envelopment Analysis. Management Science, v. 30, n .9, p. 1078-1092. DOI: 10.1287/mnsc.30.9.1078

Banker, R.D.; Charnes, A.; Cooper, W.W.; Swarts, J. e Thomas, D. (1989) An introduction to data envelopment analysis with some models and their uses. Research in Governmental and NonProfit Accounting, v. 5, p. 125-163.

DOI: $10.1007 / \mathrm{s} 11123-006-0004-8$

Barba-Romero, S. e Pomerol, J.C. (1997) Decisiones Multicriterio: Fundamentos Teóricos e Utilización Práctica. Madrid, Spain: Universidad de Alcalá.

Barros, C.P. e Athanassiou, M. (2004) Efficiency in European seaports with DEA: evidence from Greece and Portugal. Maritime Economics e Logistics, v. 6, n. 2, p. 122-140. DOI: $10.1057 /$ palgrave.mel.9100099 
Barros, C.P. (2006) A benchmark analysis of Italian seaport using data envelopment analysis. Maritime Economics and Logistics, v. 8, n. 4, p. 347-365.

DOI: 10.1057 /palgrave.mel.9100163

Bertoloto, R.F. e Soares de Mello, J.C.C.B. (2011) Eficiência de portos e terminais privativos brasileiros com características distintas. Journal of Transport Literature, v. 5, n. 2, p. 4-21.

Caillaux, M.A.; Sant'anna, A.P.; Angulo-Meza, L. e Soares de Mello, J.C.C.B. (2011) Container logistics in Mercosur: Choice of a transhipment port using ordinal Copeland method, Data Envelopment Analysis and Probabilistic Composition. Maritime Economics \& Logistics, v. 13, n. 4, p. 355-370.

DOI: $10.1057 / \mathrm{mel} .2011 .20$

Charnes, A.; Cooper, W.W. e Rhodes, E. (1978) Measuring the Efficiency of Decision Making Units. European Journal of Operational Research, v. 2, n. 6, p. 429-444.

DOI:10.1016/0377-2217(78)90138-8

Cook, W.D.; Harrison, J.; Imanirad, R.; Rouse, P. e Zhu, J. (2013) Data Envelopment Analysis with Nonhomogeneous DMUs. Operations Research, v. 61, n. 3, p. 666-676. DOI: $10.1287 /$ opre.2013.1173

Cook, W.D. e Zhu, J. (2005) Modelling Performance Measurement: Applications and Implementation Issues in DEA. New York: Springer. ISBN: 978-0-387-24137-1 (Print) 978-0387-24138-8 (Online)

Copeland, A.H. (1951) A Reasonable Social Welfare Function. Detroit, Michigan: University of Michigan.

Cortez, L.C.S.; Oliveira, L.R.D.; Martins, E.F.; Jesus, I.R.D.D. e Soares de Mello, J.C.C.B. (2012) Análise de eficiência na gestão de portos públicos brasileiros em relação ao papel das autoridades portuárias. Journal of Transport Literature, v.7, n. 2, p. 78-96. DOI: $10.1590 /$ S2238-10312013000200005

Entani, T.; Maeda, Y. e Tanaka, H. (2002) Dual models of interval DEA and its extensions to interval data. European Journal of Operational Research, v. 136, n. 1, p. 32-45. DOI: $10.1016 / \mathrm{S} 0377-2217(01) 00055-8$

Gomes, E.G.; Abreu, U.G.P.; Soares de Mello, J.C.C.B.; Carvalho, T.B. e Zen, S. (2012a) Unitary input DEA model to identify beef cattle production systems typologies. Pesquisa Operacional, v. 32, n. 2, p. 389-406.

DOI: $10.1590 / \mathrm{S} 0101-74382012005000015$

Gomes, E.G.; Soares de Mello, J.C.C.B. e Freitas, A.C.R. (2012b) Efficiency measures for a non-homogeneous group of family farmers. Pesquisa Operacional, v. 32, n. 3, p. 561-574. DOI: $10.1590 / \mathrm{S} 0101-74382012005000026$

Itoh, H. (2002) Efficiency changes at major container ports in Japan: A window application of DEA. Review of Urban and Regional Development Studies, v. 14, n. 2, p. 133-152. DOI: $10.1111 / 1467-940 X .00052$

Lovell, C.A.K. e Pastor, J.T. (1999) Radial DEA models without inputs or without outputs. European Journal of Operational Research, v. 118 , n. 1, p. 46-51. DOI:10.1016/S0377-2217(98)00338-5

Lozano, S. e Villa, G. (2009) Multiobjective target setting in data envelopment analysis using AHP. Computers \& Operations Research, v. 36, n. 2, p. 549-564. DOI: $10.1016 /$ j.cor.2007.10.015
Martinez-Budria; E., Armas, R.D.; Ibanez, M.N. e Mesa, R. (1999) A study of the efficiency of Spanish port authorities using Data Envelopment Analysis. International Journal of Transport Economics, v. 26, n. 2, p. 237-253.

Pires, L.S.; Bertoloto, R.F. e Soares de Mello, J.C.C.B. (2009) Análise da eficiência de portos de carregamento de minério de ferro. Rio's International Journal on Sciences of Industrial and Systems Engineering and Management, v. 3, n. 4, p. 094-01.

Rios, L.R. e Maçada, A.C.G. (2006) Analysing the relative efficiency of container terminals of Mercosur using DEA. Maritime Economics and Logistics, v. 8, n. 4, p. 331-346. DOI: 10.1057/palgrave.mel.9100168

Roll, Y. e Hayuth, Y. (1993) Port performance comparison applying DEA. Maritime Policy and Management, v. 20, n. 2, p. 153-161. DOI:10.1080/03088839300000025

Soares de Mello, J.C.C.B.; Gomes, E.G.; Angulo-Meza, L. e Leta, F.R. (2008) DEA advanced models for geometric evaluation of used lathes. WSEAS Transactions on Systems, v.7, n.5, p. 510-520.

Soares de Mello, J.C.C.B.; Gomes, L.F.A.M.; Gomes, E.G. e Soares de Mello, M.H.C. (2005) Use of ordinal multi-criteria methods in the analysis of the Formula 1 World Championship. Cadernos Ebape.BR, v. 3, n. 2, p. 1-8. DOI: $10.1590 / \mathrm{S} 1679-39512005000200004$

Tongzon, J. (2001) Efficiency measurement of selected Australian and other international ports using data envelopment analysis. Transportation Research Part A: Policy and Practice, v. 35, n. 2, p. 107-122. DOI: 10.1016/S0965-8564(99)00049-X

Turner, H.; Windle, R. e Dresner, M. (2004) North American container port productivity. Transportation Research Part E: Logistics and Transportation Review, v. 40, n. 4, p. 339-356. DOI: $10.1016 /$ j.tre.2003.06.001

Valentine, V. C. e Gray, R. (2001) The measurement of port efficiency using data envelopment analysis. In Proceedings of the Ninth World Conference on Transport Research, South Korea: Seoul.

Yamada, Y.; Matui, T. e Sugiyama, M. (1994) New analysis of efficiency based on DEA. Journal of the Operations Research Society of Japan, v. 37, n. 2, p. 158-167. DOI: $10.1007 / 978-3-642-16530-620$. 